\title{
Elaboración de un plan maestro logístico en una empresa de la industria de jabones
}

\author{
Development of a logistics master plan in a soap industry company
}

\author{
A. Brand-Tejeda ; ; F. A. Mejía-Espinal iD; A.M Paredes-Rodríguez iD ; N. Arias-Brito iD
}

\begin{abstract}
Resumen - El plan maestro logístico es un modelo de análisis de un determinado sector de una organización que incluye el diagnóstico, investigación, análisis y propuesta. El presente trabajo contiene la aplicación y aproximación de un plan maestro logístico para una PYME ubicada en Cali, Colombia. La empresa Caso de Estudio está dedicada a la producción y distribución de jabón de loza. Dicho esto, en este proyecto se buscó estructurar un modelo de gestión integral de sus procesos de abastecimiento, producción y distribución, que permita que la organización sea más competitiva en un mercado hoy globalizado. Se hace uso de modelado de negocios con el fin de esquematizar el presente de la entidad, para determinar las actividades que no generan valor a la misma y por ende poder of recer propuestas de mejoramiento en dichos procesos. Entre los principales resultados obtenidos se encuentran la separación que existe entre la logística de abastecimiento, logística de producción y logística de distribución como islas de información, las cuales funcionan desarticuladamente.
\end{abstract}

Palabras claves - Abastecimiento, Distribución, Gestión Integral, IDEFo, Plan Maestro, Producción.

\begin{abstract}
The logistics master plan is a model of analysis of a certain sector of an organization that includes the diagnosis, investigation, analysis and proposal. This work contains the application and approach of a logistics master plan for an SME located in Cali, Colombia. The Case Study is a company dedicated to the production and distribution of earthenware soap. That said, this project sought to structure a comprehensive management model of its supply, production and distribution processes, allowing the organization to be more competitive in a globalized market today. Business modeling is used to outline the present of the entity, to determine the activities that do not generate value to it and to be able to offer proposals for improvement in said processes. Among the main results found are the separation that exists between supply logistics, production logistics and distribution logistics as information islands, which operate disjointedly.
\end{abstract}

Index terms - Supply, distribution, Integral Management, IDEF0, Master plan, production.

Este manuscrito fue enviado el 02 de julio de 2019 y aceptado el 26 de marzo de 2020. Este documento fue enviado el 17 de mayo del 2020 y fue aceptado el 29 de septiembre del 2020.

A Brand-Tejada estuvo en la Corporación Universitaria Minuto de Dios, sede Buga, Calle 21 sur Carrea 12 (abrandtejed@uniminuto.edu.co)

F. Mejía está ahora en Universidad Autónoma, sede Cali, Calle 25, Vía

Cali - Puerto Tejada \#\#115-85 Km 2, Jamundí. (famejia@uao.edu.co)

\section{INTRODUCCIÓN}

$\mathrm{E}^{\mathrm{n}}$ n la actualidad, las estrategias logísticas son de vital importancia para el trabajo de las organizaciones: el conocimiento de las necesidades y expectativas en la relación clientes y proveedores, es sumamente importante para reducir los plazos de entrega y conseguir la satisfacción del cliente. En palabras de Ballou: "la logística gira en torno a la creación de valor: valor para los clientes, los proveedores y los accionistas de la empresa" [1]. La gestión logística debe ser llevada a cabo de tal forma que se controlen los costos de operación, dichos costos se pueden agrupar en: transporte, almacenamiento, inventario y administración. Para garantizar que la logística dentro de las áreas de abastecimiento, producción y distribución cumplan con los requisitos de los clientes, deberá ser competitiva, flexible y proactiva. El propósito general de una organización eficiente, deberá ser la mejora continua de la productividad y rendimiento.

Como precedentes se tienen el trabajo [2] permite identificar los procesos de orden logístico, relacionados con la gestión de la cadena de abastecimiento, por lo que facilitó la ejecución del plan maestro logístico. Similarmente, [3] dictamina que la globalización ha transformado al mundo en un mercado más heterogéneo y con ciclos de vida más cortos de los productos. [4] presenta una propuesta de implementación de un modelo de Green Supply Chain Management (GSCM) para una empresa comercializadora de suministros eléctricos en un contexto globalizado.

En esa línea [5] presentan el estudio de los sistemas de indicadores de desempeño de cadenas de abastecimiento en ambientes de incertidumbre. Por otra parte, en [6] estudian y ofrecen una herramienta para el mejoramiento en la gestión de inventarios de la empresa Industria Saga S.A.S. [7] presenta la ejecución de un plan maestro con objetivos de seguridad urbana en Chile.

En [8] se propone un procedimiento para la elaboración de mapas de procesos de servicios hospitalarios. [9] permitirán apoyar diagnósticos organizacionales en cualquier laboratorio de metrología regido por la Norma Técnica Colombiana NTC

A.M Paredes-Rodríguez está ahora en Corporación Universitaria Minuto de Dios, sede Buga, Calle 21 sur Carrea 12 (aparedesrod@uniminuto.edu.co)

N. Arias-Brito está ahora en Corporación Universitaria Minuto de Dios, sede Buga, Calle 21 sur Carrea 12 (norbey.arias@uniminuto.edu) 
17025. También [10] realiza un análisis de varias propuestas encaminadas a mejorar el proceso de desarrollo de software en Cuba.

En [11] se brindan pautas para identificar aspectos de mejora en el modelado de los procesos de abastecimiento, producción y distribución de la empresa Caso de Estudio. En [12] evaluaron la planificación y el control de la gestión de abastecimiento en la empresa Caso de Estudio. [13] generaron un enfoque relacionado con la construcción de estado del arte para procesos de gestión logística. [14] analizaron el comportamiento de la demanda a lo largo de la cadena de suministro y conocer la importancia de la sincronización y coordinación entre los canales de distribución. De tanto, [8]cuestionan factores relacionados con el cumplimiento de pedidos, comercialización de los productos de la organización, la gestión de inventarios, entre otros.

En [15] se describe el desarrollo de un aplicativo computacional para la planeación y secuenciación de la producción en una empresa colombiana fabricante de autopartes. [16] generó un enfoque relacionado con el modelado de proceso en PYMES, aplicando herramientas lean. [17] permitió conocer información de las herramientas de la manufactura esbelta y analizar la implementación del mapa de la cadena de valor en la empresa Caso de Estudio. En [18] brindó la metodología de implementación del mapa de la cadena de valor en la empresa Caso de Estudio.

El plan maestro planteado pretendió visualizar las operaciones núcleos de la organización y los actores rectores que intervienen en las mismas, bajo un lenguaje simple en entornos complejos con la aplicación de herramientas tecnológicas que den claridad y diagramen la actualidad del proceso.

\section{PLANTEAMIENTO DEL PROBLEMA}

El sector productor de cosméticos y de productos de aseo presenta una estructura de mercado oligopólica en la cual ta competencia radica en estrategias de diferenciación de producto cuyo objetivo es capturar nichos de mercado. Frente a las exigencias crecientes de los consumidores, las tendencias en moda y la competencia internacional, las estrategias de las empresas del sector se basan en la segmentación de mercados y la constante depuración de su portafolio de marcas. Con esto las firmas buscan adaptarse a los cambios rápidos del mercado y disminuir el riesgo de acumular inventarios y producir bienes obsoletos. Al igual que en el segmento de productos para el cuidado personal, hay una alta sustitución entre marcas que en periodos de contracción favorece a líneas más económicas. La capacidad para capturar mercados mediante estrategias publicitarias y diferenciación de productos es relativamente mayor en el caso de las firmas de capital extranjero, hecho que se refleja en su liderazgo en la participación en el mercado.

La empresa objeto de estudio actualmente cuenta un proceso de abastecimiento, producción y distribución, con el cual logra la fabricación de productos del sector cosmético como lo es el jabón.
Para determinar el estado actual del proceso logístico de la compañía se utilizó la elaboración de un mapa de flujo de valor (o más conocido como VSM).

Luego de la aplicación de la metodología se logra detectar que no existe planeación ni control de la producción y el direccionamiento de toda la organización depende completamente de la presencia y decisiones de la gerencia, pues es esta la encargada activar, la compra de materias primas, la producción y/o el despacho del pedido a los clientes. Esta decisión genera un aumento excesivo de tiempo de entrega de los pedidos, lo que genera retrasos en los tiempos de respuesta. El pedido llega a la empresa es tomado por la gerencia, quienes se encargan de emitir la orden de producción, despacho de pedido y de compra de materias primas según sea necesario. Es decir, una vez se toma el pedido del cliente, se verifica la existencia de producto terminado para ordenar su despacho. En el evento en que no existan unidades en el almacén de producto terminado, la gerencia emite la orden de producción. El área de producción se encarga de tomar las cantidades necesarias de las materias primas para suplir la orden emitida (tiempo promedio 4,26 minutos por lote).

En caso de que falte materia prima para dicho requerimiento, se reporta a gerencia la inexistencia de materias primas, esta procede a establecer contacto con el proveedor y emite la orden de compra de lo requerido. El tiempo promedio de llegada del pedido de las materias primas, una vez se haya hecho entrega del comprobante de pago es de 2,7 días (3888 minutos).

El proceso de fabricación de jabón de loza está constituido por 4 etapas, como son:

Mezclado: es un proceso realizado por un operario en el cual se añaden los insumos equivalentes a la producción de lotes de 20 unidades de jabón de $500 \mathrm{~g}$, la cual, en un periodo de tiempo promedio de 22,464 minutos por lote, da consistencia y homogeneidad a la mezcla.

Moldeado: Una vez se termina el proceso de mezclado, el operario toma la muestra y la traslada a la estación de moldeo (tiempo promedio de traslado de 1,3 minutos por lote) donde demora 33,618 minutos en moldear las 20 unidades de jabón.

Aireado: el aireado es un periodo de secado de 48 horas promedio (2880 minutos) que se le da al jabón para evitar que resulte con ruptura y/o fracturas en los procesos de empaque y distribución.

Empaque: en este proceso se le coloca la etiqueta y la envoltura al jabón de loza y posteriormente se llevan al almacén de producto terminado. El operario de empaque demora en promedio 10,3 minutos por lote, con un tiempo promedio de traslado y deposito en el almacén de producto terminado de 3,04 minutos por lote.

El máximo tiempo de entrega del pedido una vez emitida la orden de despacho es de 31,21 minutos (considerando el cliente que se encuentra a mayor distancia), con un tiempo promedio de alistamiento de mercancía de 5,07 min/pedido.

Debido a la ausencia de un proceso de planeación y control estratégico, se hace necesario la elaboración de un plan maestro logístico que gestione integralmente los procesos de abastecimiento, producción y distribución de jabón de loza. 


\section{RESULTADOS}

1. El modelo propuesto tiene como base la visualización que conllevan las actividades, analizando los procesos claves, en el cual se corregirán las actividades presentadas en el estado actual del proceso de fabricación de jabón de loza en la búsqueda de añadir valor en los tiempos de respuesta del sistema, costos y procesos.

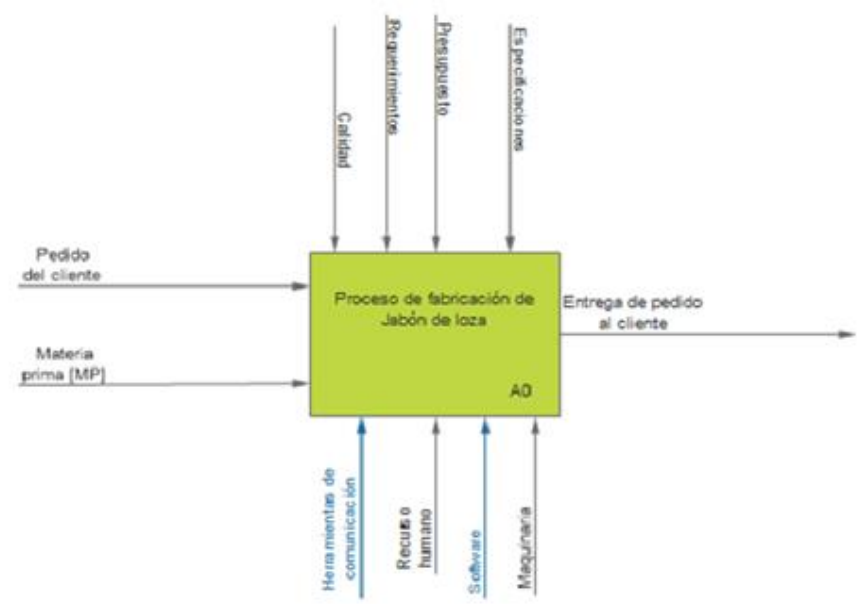

Fig. 1. Modelo Integral del Plan Maestro para la Producción de Jabón de Loza.

2. Se propone la optimización de la comunicación con los clientes haciendo uso de las facilidades tecnológicas de comunicación, las cuales permiten una conexión directa con otras organizaciones; también la implementación de software de apoyo para el control gerencial e informativo de los inventarios (Como puede evidenciarse en Fig.1), de manera que estos dejen de ser una restricción del sistema como lo son actualmente. En términos generales, es preciso resaltar la importancia de llevar registro de los datos de producción, demanda, participación de cada material en la producción de cada unidad de producto, como también la participación de cada línea de producto en las ventas. A partir de lo anterior, se recomienda hacer uso de herramientas de gestión de operaciones de enfoque estratégico, táctico y operático, con el fin de administrar de manera eficiente los recursos de la organización, articular las diferentes áreas operativas y eliminar los tiempos muertos, retrasos en los pedidos y demoras por producción a causa de actividades ociosas como son:

3. La planeación agregada, con el propósito de conocer la capacidad de la planta (potencial productivo, layout, potencial humano, inventarios y/o tecnologías) y determinar la estrategia de fabricación que optimiza los recursos y minimiza los costos de producción en la compañía.

4. El plan maestro de producción, es importante para la organización realizar una planeación más detallada, determinar que hacer y cuando hacerlo, en conformidad con el plan agregado definido anteriormente. Dicho esto, se recomienda estudiar el mercado objetivo de la organización (muchos útiles, pocos vitales) y en lo posible realizar alianzas y/o contratos con nuestros clientes que permitan definir el horizonte de planeación y establecer ordenes en firme.
5. La planeación de requerimiento de materiales, dada la importancia de conocer que necesitamos para producir, cuando lo necesitamos producir y cuando ordenar la producción y la compra de los materiales, ejecutar una administración optima de los inventarios y la programación de los pedidos de abastecimiento según el sistema de loteo que minimice los costos de mantener y ordenar.

6. La implementación de bases de datos actualizadas permitiría suministrar en tiempo real información para los planes de producción y el manejo de inventarios. Dicho esto, se elimina la incertidumbre de la materia prima existente actualmente en almacén, la cantidad de producto existente para atender el pedido del cliente. En la parte de producción, se propone la construcción de cartas de procesos de manera que estos puedan ser estandarizados y se elimine la dependencia que existe del operador en el modelo actual.

7. En el proceso de abastecimiento, se propone afianzar las relaciones con los clientes de manera que se lleguen a acuerdos que aumenten la eficiencia de los procesos de transacción y envío; también se recomienda la implementación de software de control de los inventarios de materias primas y producto terminado, para con esto brindar mayor eficacia en la respuesta al pedido del cliente, la utilización eficiente del presupuesto de abastecimiento de materias primas, los sobrecostos por concepto de pedido de mercancías, y la optimización del transporte de las mismas.

Con el modelo de abastecimiento planteado, se busca eliminar la demora generada por la consignación y posterior envío de la factura de pago para poder empezar el alistamiento de la materia prima por parte del proveedor (lo que generaba uno o dos días de retraso, dependiendo el día de consignación), se propone un sistema de abastecimiento que no dependa del informe de inexistencias de materias prima actualmente generado por el área de producción. El concepto de fidelización con los proveedores, está relacionado con la construcción de nexos fiables entre la empresa Industrias Zagas S.A.S y los mismos de manera que estos brinden la mayor disponibilidad posible de materias primas atendiendo a la planeación táctica y operativa establecidas en el plan maestro de producción y el plan de requerimiento de materiales (anteriormente recomendados).

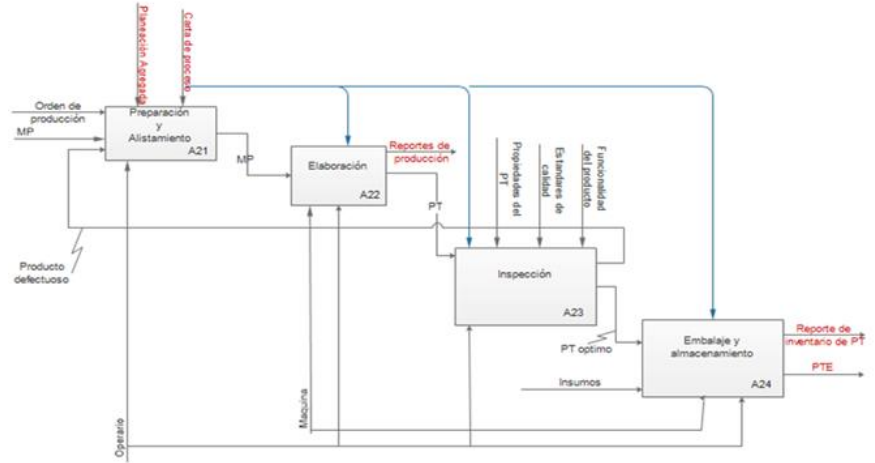

Fig. 2. Modelo de Producción Integral para el Plan Maestro en la Fabricación de Jabón de Loza.

8. Se recomienda estandarizar los procesos de producción para eliminar las restricciones ligadas a los inventarios de producto terminado, volumen de producción, producción según las especificaciones de las materias primas que llegan a planta, y al 
embalaje según el volumen del pedido. Se propone que el área de distribución y ventas (de manera articulada) se encarguen de recibir directamente el pedido del cliente y al existir producto terminado (dado el sistema de gestión propuesto) se obtenga mayor nivel de servicio al cliente y por ende mayor satisfacción de los mismos. Dicho esto, se eliminan las excepciones y/o prioridades debidas a los horarios de recibo de mercancías de los clientes (ver Fig.2). Y es el jefe de despachos quien emite la orden de distribución con toda la información necesaria para la efectiva entrega del pedido, es decir, ruta, ubicación, horario de entrega, volumen de pedido, flota necesaria, tiempo estimado de recorrido, costo de envío según la distancia a recorrer, entre otros), como también será el encargado de definir el medio de pago del mismo.

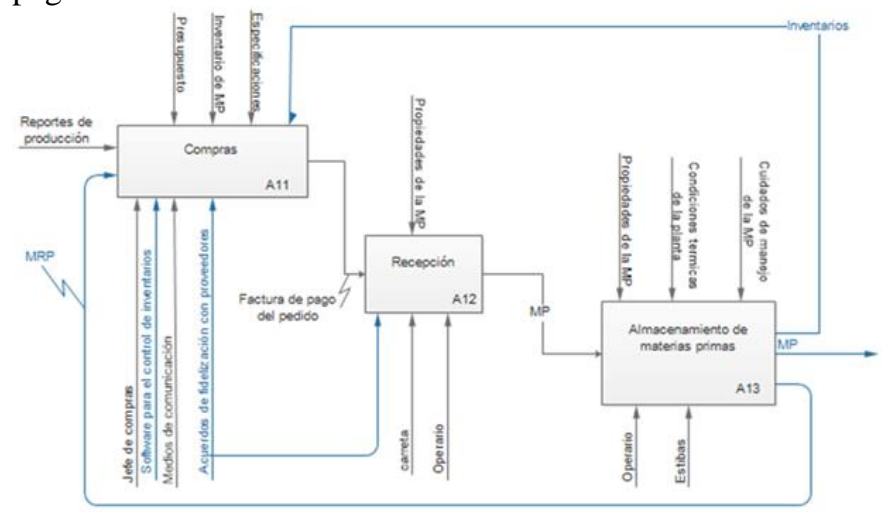

Fig. 3. Modelo de Abastecimiento Integral para el Plan Maestro en la Fabricación de Jabón de Loza.

9. El modelo de gestión integral de abastecimiento, producción y distribución propuesto (Fig.3), busca aumentar la eficiencia en los procesos y procedimientos a lo largo de la cadepa de suministro, asignando a cada área tareas concretas de gerenciamiento, como son órdenes de compra, de producqión y de despacho, las cuales actualmente dependen del gerente de la empresa y de la existencia de material y producto terminado. Se recomienda la implementación de un inventario de seguridad, con el fin de generar una existencia mínima de materia prima y de producto terminado para tener un margen de operaciṕn tal que no se generen retrasos en la entrega de pedidos al cliente. A partir de la comparación del modelo actual con la propuesta de un modelo de mejoramiento donde se modifican y/o eliminan las actividades que no están generando valor a la gestión integral de los procesos, se presentan los impactos esperados una vez se apliquen dichos cambios; lo anterior, en busca del mejoramiento de los procesos productivos y el aumento de la competitividad de la organización.

En pro del mejoramiento del nivel de servicio y la respuesta al cliente se recomiendan los siguientes cambios:

1. Llevar registro del comportamiento de la demanda, con base a estos pronosticar y/o prever el comportamiento de la misma. Lo que permitirá realizar una planeación estratégica, táctica y operativa en la organización.

2. El pedido lo tome el departamento de distribución y ventas con el propósito de responder eficazmente al cliente.
3. La producción se determine por el MPS definido para cada línea de producto.

4. La compra de materias primas sea administrada por el área de abastecimiento, apoyada en el MRP establecido, a partir de los pronósticos, el plan agregado y el plan maestro de producción.

5. Establecer un inventario de seguridad de materias primas, producto en proceso y producto terminado. Dicho esto, se elimina las demoras sujetas a los trámites y transporte de la materia prima, al proceso de aireación y de emisión de órdenes de compra, producción y despacho.

6. Implementar un almacén de producto terminado en el cual se realiza el proceso de aireación.

7. Unir los procesos de mezclado y moldeado en una misma estación, de modo que el operario no tenga que desplazarse con la materia prima.

Simulación de costos de producción con la propuesta de mejoramiento

A partir de la propuesta de mejoramiento y contemplando un nivel de inventario de seguridad de 400 unidades (teniendo en cuenta que la infraestructura de la planta no permite el almacenamiento de una mayor cantidad de inventario de producto terminado), se plantea un plan de producción, con la fuerza laboral, los costos directos e indirectos de fabricación como se encuentran actualmente. Esta simulación se realizará teniendo en cuenta tres escenarios de comportamiento de la demanda, con una estrategia de nivelación de producción, es decir, un plan de producción con fuerza laboral constante y horas extras a seis meses vista sobre la producción de jabón de loza en su presentación de $500 \mathrm{~g}$

Demanda real, hace referencia al comportamiento real de la demanda de la empresa industrias Sagaz S.A.S.

Enfoque y/o proyección pesimista, se refiere a una proyección en la cual se esperará que la demanda real disminuye en un $25 \%$. Esto nos permite evaluar el comportamiento y repercusiones que tiene este hecho sobre la utilización del recurso humano, maquinaria y los costos.

Enfoque y/o proyección optimista, es la proyección optimista de la producción, es decir, evaluar y analizar los efectos que lleva consigo el aumento de la demanda en un $70 \%$. A continuación, se muestra la información de costos tenida a día 10 de noviembre de 2014:

Los costos de los materiales de fabricación suman un total de $\$ 86786,08$. En cuanto a los materiales indirectos de fabricación se calculó un costo de $\$ 13.764,33$ y los costos indirectos de la fabricación un total de $\$ 66.968224$. Frente a los costos de los materiales directos de fabricación de jabón de loza $500 \mathrm{~g}$, para $82,95 \mathrm{Kg}$ de jabón, ascienden a un total de \$109.208,16. Los costos por hora de la fabricación de jabón ascienden a un total de $\$ 19628$ por hora.

El costo total unitario en la fabricación de jabón de loza en la presentación de $500 \mathrm{~g}$, así: el costo por kilogramo de jabón de loza por concepto de materiales directos de fabricación es de \$1317. Dicho lo anterior, el costo de los materiales indirectos de fabricación es de $\$ 60,50$. Los costos de jabón de loza por concepto de materiales directos es de $\$ 1317$, la mano de obra 
directa cuesta $\$ 237$ y el costo total unitario del jabón de loza en la presentación de $500 \mathrm{~g}$ es de $\$ 837,09$. De la información presentada, se plantea un plan con fuerza laboral constante y utilización de horas extras que no requiere de la utilización de horas extras puesto que la capacidad de producción actual no genera unidades faltantes. Inicialmente había tiempo ocioso y/o la utilización de la mano de obra no era óptima. No obstante, los últimos meses, dado al aumento en la demanda del producto, la utilización ha aumentado llegando a su tope máximo. En ese sentido, los costos totales del plan logístico en situación real generan \$9'900.000 pesos por concepto mano de obra y $\$ 720.000$ por concepto de almacenamiento, para un total de $\$ 10$ '620.000 pesos.

El registro de demandas con un enfoque pesimista muestra una disminución de la demanda en un $25 \%$. Dado esto, no se requiere de la utilización de horas extras puesto que la capacidad de producción es mucho mayor a la demanda y no se generan unidades faltantes. Sin embargo, se genera un porcentaje de utilización de la fuerza laboral muy bajo. El costo total del plan logístico de enfoque pesimista genera $\$ 9^{\prime} 900.000$ pesos por concepto mano de obra y $\$ 720.000$ por concepto de almacenamiento, para un total de $\$ 10$ '620.000 pesos.

En cuanto al registro de demandas con un enfoque optimista (aumento de la demanda actual en un 70\%). Dado esto se puede decir que se requiere de la implementación de otra línea de producción, puesto que con la actual no se satisface la demanda. Dado que el tope máximo de horas extras son 100 horas mensuales (a 25 días por mes) por línea de producción, se generan demasiadas unidades faltantes para satisfacer la demanda. Los costos totales del plan logístico con enfoque optimista generan $\$ 41^{\prime} 465.465$ pesos en pago de horas extras, $\$ 9 ' 900.000$ pesos por concepto de mano de obra con horas regulares de trabajo, $\$ 807.540$ pesos en almacenamiento de producto terminado y $\$ 3$ '876.192 pesos por concepto de unidades faltantes, para un total de 54'434.117 pesos.

\section{CONCLUSIONES}

La documentación de referentes, permite conocer el estado actual del tema a desarrollar, brindando un direccionamiento puntual a la construcción metodológica de los componentes de un plan maestro logístico, donde sea concerniente para estudios o propuestas futuras. El desarrollo del estado del arte, permitió identificar el poco material o referentes de un plan maestro logístico, por ende, se construye a partir de ponencias, fases y procesos de abastecimiento, producción y distribución, con un desarrollo práctico y aplicativo en un área, sector o empresa específica, mediante herramientas basadas en la visualización e interoperabilidad de los procesos, desarrollo base de la ingeniería e integración empresarial.

La coordinación de las actividades relacionadas con la logística siempre pretende añadir valor a los productos y servicios para lograr la satisfacción del cliente. Muchas de las PYME no cuentan con una estrategia de distribución definida, ni flujo de información adecuada con los diferentes clientes, afectando la planeación logística en el momento de un requerimiento. Desarrollar un plan maestro logístico, constituye una ventaja competitiva para cualquier empresa, en la medida que afecte positivamente aspectos tales como: la reducción de costos, reducción de capital, mejora de servicios, eliminación de operaciones que no agregan valor. Es de vital importancia tomar las decisiones correctas para alcanzar esa ventaja competitiva a partir de entender el comportamiento sistemático de la organización y del mercado.

La logística en la empresa no se ejecuta como se define en el marco teórico con base al concepto de sistema, desde el proveedor hasta el cliente final, presenta una concepción errada de la misma, donde la logística se centra en el área de distribución de producto final; existe una separación entre la logística de abastecimiento, logística de producción y logística de distribución como islas de información, las cuales funcionan desarticuladamente. La cadena de suministro integra los procesos del negocio desde el proveedor hasta el consumidor [19] La industria objeto de estudio, no está configurada para promover la eficiencia, encontrándose rupturas en el flujo de información entre los eslabones del proceso. El tener conocimiento sobre los factores externos es lo que permiten establecer la capacidad competitiva de la empresa en relación con sus recursos internos. El modelo propuesto incluye todas las variables de entrada necesarias para realizar una gestión de compras de materias primas eficiente, que, sumado a la efectiva comunicación entre los diferentes procesos, evitará que el subsistema de abastecimiento genere problemas de atrasos, paradas de planta e incumplimiento a los clientes. El éxito de las estrategias a implementar está directamente relacionado con la calidad de información, que los estrategas asimilen de la organización y su entorno, al igual que su capacidad creativa para sortear las condiciones del mercado. El modelo mejorado propuesto para Industrias Sagaz, permite optimizar tiempos y eliminar actividades que no generan valor a lo largo de la cadena de suministro, evitando reprocesos y mejorando la competitividad de la empresa.

\section{RECOMENDACIONES}

Establecer los costos por cada kilómetro de transporte de mercancía hacía los clientes, puesto que este está directamente relacionado con el deterioro, consumo de combustible, utilización y mantenimiento del vehículo de transporte. Enfocar esfuerzos en aumentar la demanda, apoyados en el área de mercadeo y ventas. Se recomienda tener en cuenta la base teórica sobre la que se estructuró el proyecto, ya que el estado del arte muestra hasta donde han avanzado las investigaciones describiendo el nivel más alto conseguido hasta el momento en este campo, brindando herramientas fundamentales como modelos, sistemas, programas, técnicas, tendencias, etc., para formular la solución del problema.

Realizar acuerdos de fidelización con los proveedores para optimizar los tiempos de procesos legales y activar el envío de las materias primas de forma inmediata. Establecer un sistema de manejo de inventarios que permita aumentar la productividad y competitividad de la organización. Eliminar la dependencia directa de gerencia para la emisión de las órdenes de producción, compra y despacho. Dicho esto, se recomienda independizar y/o sectorizar estas según la necesidad que evidencien los jefes de cada área. Diseñar, estandarizar y documentar procesos y procedimientos dentro de la 
organización, tanto a nivel operacional como administrativo. La simulación de los procesos y subprocesos permite experimentar diferentes opciones para superar las restricciones, brindando información confiable y disminuyendo así la probabilidad de cometer errores, ya que las decisiones se fundamentan en resultados precisos y planificados.

Desarrollar registros de demanda, producción, despachos, inventarios, compras y contabilidad. Desarrollar herramientas de Lean manufacturing con el objeto de inculcar cultura organizacional y mejoramiento continuo. Se recomienda a la empresa implementar el plan maestro logístico, dado que es una herramienta fundamental para lograr la estandarización y el mejoramiento de los procesos y procedimientos, en busca de superar las expectativas de los clientes. Se debe diseñar e implementar un sistema de información, el cual arroje resultados en tiempo real, y permita tomar decisiones antes de incumplir con los requerimientos establecidos por los clientes internos y externos.

\section{REFERENCIAS}

R. H. Ballou, Logistica Administración de la cadena de suministro. 2004.

[2] M. Barón, I. Huertas, and J. A. Orjuela Castro, Gestión de la cadena de abastecimiento del biodiésel: una revisión de la literatura, vol. 18 , no. 1. 2013.

[3] O. Aguilar Rascón, R. Posada Vasquez, and M. S. Soto Sevilla, "E1 otro lado de la logística, una visión del aprovisionamiento en estratégica: tendencias las cadenas de valor para el desarrollo sostenible," Rev. Cent. Investig., vol. 53, no. 9, pp. 221-232, 2012, doi: 10.1017/CBO9781107415324.004.

[4] R. Gómez Montoya, "Propuesta de gestión de cadena de abastecimiento verde para empresa comercializadora de suministros eléctricos," Prod. + Limpia, vol. 6, no. 2, pp. 117-127, 2011.

[5] M. D. Arango Serna, W. Adarme Jaimes, and J. A. Zapata Cortes, "Gestión cadena de abastecimiento - logística con indicadores bajo incertidumbre, caso aplicado sector panificador Palmira," Cienc. $e$ Ing. Neogranadina, vol. 20, no. 1, p. 97.115, 2010.

[6] V. Gutiérrez and D. P. Jaramillo, "Review of the software available for inventory management in supply chains in Colombia," Estud. Gerenciales, vol. 25, no. 110, pp. 125-153, 2009, doi: 10.1016/S0123-5923(09)70065-8.

[7] I. Cartes, "Plan maestro de reconstrucción de Dichato: del sitio cero a las plataformas de futuro," Arquit. del Sur, vol. 38, pp. 38-51, 2010.

[8] A. Hernández, A. Medina, and D. Nogueira, "Criterios para la elaboración de mapas de procesos. Particularidades para los servicios hospitalarios.," Ing. Ind., vol. 30, no. 2, p. 1, 2009, [Online]. Available: https://www.redalyc.org/articulo.oa?id=360433569002.

[9] E. Diaz Benachi, C. C. Rivera Muñoz, S. I. Escobar Rodríguez, and O. A. Rojas Alvarado, "Modelado de los procesos de gestión para laboratorio de metrología del sector energético," Rev. Gerenc. tecnológica informática., vol. 13, pp. 79-94, 2014.

[10] N. Silega, D. Macías, J. P. Febles, and M. Noguera, "Estado de la complejidad arbitraria y Arquitectura Dirigida por Modelos en el desarrollo de software en Cuba.," State Arbitr. Complex. Model Driven Archit. Softw. Dev. Cuba., vol. 8, no. 1, pp. 156-171, 2014, [Online]. Available: http://search.ebscohost.com/login.aspx?direct=true \&db=fua\&AN=9 4967097\&lang=es\&site=ehost-live.

[11] F. Alarcón-Valero, M. D. M. Eva-Alemany Díaz, and F. JeanpierreLaguardia, "Metodología para la reingeniería basada en el modelado de procesos de negocio," Dyna, vol. 87, no. 5, pp. 566-573, 2012, doi: $10.6036 / 4585$.

[12] C. A. P. Pérez and C. A. López Bello, Una revisión al estado del arte de la integración de toma de decisiones en la red logística, vol. 18, no. 1.2013.

[13] V. Sanchez and Z. H. Vianch, "Revista Científica Ingeniería y
Modelos y configuraciones de cadenas de suministro en productos perecederos," Rev. Cient. Ing. y Desarro., vol. 32, no. 1, pp. 1-8, 2019.

[14] Marco Alberto Valenzo-Jiménez; Evaristo Galeana-Figueroa; Jaime Apolinar Martínez-Arroyo, "Competitividad y supply chain management en la industria exportadora de aguacates en méxico," Rev. Int. Adm. Finanz., vol. 8, no. 2, pp. 1-20, 2015, [Online]. Available: http://www.theibfr.com/ARCHIVE/RIAF-V8N22015.pdf.

[15] A. Hernández and G. Mejía, "Aplicativo computacional para la planeación de la producción en una empresa fabricante de autopartes Computer Application for Production Planning and Scheduling of an Auto Part Company in Colombia," Rev. Iberoam., pp. 24-31, 2008.

[16] O. L. M. Celis and J. M. S. García, "A technological approach to the development of logistic projects using 'Lean Six Sigma," Estud. Gerenciales, vol. 28, no. 124, pp. 23-43, 2012, doi: 10.1016/S01235923(12)70214-0.

[17] J. G. Arrieta Posada, J. D. Muñoz Dominguez, A. Salcedo Echeverri, and S. Sossa Gutiérrez, "Aplicación Lean Manufacturing En La Industria Colombiana," Ninth LACCEI Lat. Am. Caribb. Conf. (LACCEI'2011), Eng. a Smart Planet, Innov. Inf. Technol. Comput. Tools Sustain. Dev. August 3-5, 2011, Medellin, Colomb., pp. 1-11, 2011.

[18] F. A. Abdulmalek and J. Rajgopal, "Analyzing the benefits of lean manufacturing and value stream mapping via simulation: A process sector case study," Int. J. Prod. Econ., 2007, doi: 10.1016/j.ijpe.2006.09.009.

[19] D. M. Lambert, "The Eight Essential Supply Chain Management Processes," Supply Chain Manag. Rev., vol. 8, no. 6, pp. 18-26, 2016.

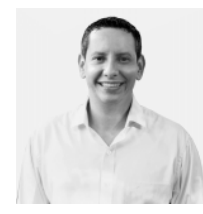

Alvaro Hernan Brand-Tejeda, nacido el 31 de enero de 1983, Buga, Valle del Cauca, Colombia. Ingeniero Industrial, Magister en Logística Integral de la Universidad Autónoma de Occidente, Santiago de Cali, Valle del Cauca, Colombia. Actualmente Secretario de Turismo y Desarrollo Económico, Buga, Valle del Cauca, Colombia, docente instructor 2 en la Corporación Universitaria Minuto de Dios, Buga, Valle del Cauca.

ORCID: http://orcid.org/0000-0001-5580-8726

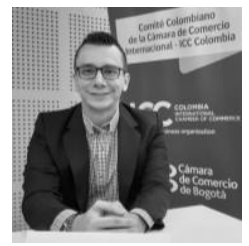

Fabian Andres Mejia-Espinal, nacido el 29 de enero de 1985 en Tuluá, Valle del Cauca, Colombia. Administrador Empresarial, Trainer on Incoterms ${ }^{\circledR} 2020$ por la Cámara de Comercio Internacional Colombia, Magister en Logística Integral de la Universidad Autónoma de Occidente,

Candidato a Doctor en Ciencias Económicas y Administrativas de UCIMEXICO. Actualmente presidente de PROAGRO y Profesor en Logística Internacional \& Negocios Internacionales de la Universidad Autónoma de Occidente e ICESI.

ORCID: http://orcid.org/0000-0002-7009-1695 


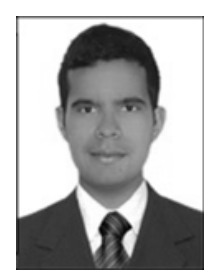

Andres Mauricio Paredes-Rodríguez, nacido el 25 de febrero de 1994 en San Pedro, Valle del Cauca, Colombia. Ingeniero Industrial, estudiante de Maestría en Ingeniería Industrial de la Universidad del Valle, Valle del Cauca, Colombia. Docente tiempo completo del Programa de Ingeniería Industrial de la Corporación Universitaria Minuto de Dios, Buga, Valle del Cauca.

ORCID: http://orcid.org/0000-0001-9196-9965

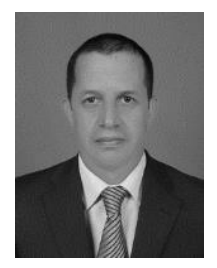

Norbey Arias-Brito, nacido el 21 de agosto de 1966 en Pereira, Risaralda, Colombia. Administrador de Empresas, Especialista en Gerencia de Logística, Magister en Administración de la Universidad Nacional de Palmira, Valle del Cauca, Colombia. Docente tiempo completo del Programa de Ingeniería Industrial de la Corporación Universitaria Minuto de Dios, Buga, Valle del Cauca.

ORCID: http://orcid.org/0000-0003-1284-8255 